\title{
How Late Can First Births Be Postponed? Some Illustrative Population-level Calculations
}

\author{
Joshua R. Goldstein*
}

\begin{abstract}
I shift, stretch, and transform the observed cohort age-schedule of first birth for Danish women born in 1963 to see how late the mean age at first birth could plausibly shift. Constraints of two kinds are placed on the ultimate distribution of first births. First, no more than one-third of first births can occur after age 35. This constraint allows postponement without radical changes in childlessness or parity distribution. Second, I preserve some variability in the age at first birth by keeping the standard deviation of first birth above 4 years, the minimum value observed for Denmark during the baby boom years. Under these constraints, I find that mean ages at first birth of at least 33 years are plausible. This would represent a further increase of about 4 years in the mean age at first birth seen in recent periods. I conclude that the depressed levels of fertility seen due to postponement could continue for decades before limits are reached.
\end{abstract}

\section{Introduction}

Postponement, measured as increases in mean age at childbearing, cannot continue forever given a fixed age-range of childbearing.

Hagewen and Morgan (2005)

Childbearing has shifted to older ages throughout the industrialised world. Most observers believe that the postponement of childbearing is a transitional process, which must come to an end. ${ }^{1}$ On the other hand, the forces that have lead to postponement, including expanding educational attainment, high young adult

\footnotetext{
Joshua R. Goldstein, Office of Population Research, Princeton University, Princeton NJ 08544, USA. Email: josh@princeton.edu

1 Indeed, in public discussion at the conference on Postponement of Childbearing in Europe, held in Vienna, Austria, 1-3 December 2005, John Bongaarts predicted that postponement would stop within 10 years.
} 
unemployment, and long partnership searches show few signs of slackening. In this note, I try to provide an answer to how much longer postponement could continue before running into biological and social limits. I use illustrative scenarios to calculate plausible upper limits for the mean age at first birth for populations given present reproductive technology and assuming that there will continue to be considerable variation in the timing of first birth within the population. I find, using conservative assumptions, that first-birth schedules with a mean age at of 33 to 34 years are plausible.

\section{Background and Importance}

The postponement of first births has been going on for about three decades in most European countries. It began in Scandinavia in the early 1970s, in continental Europe in the mid-1970s, and in the Mediterranean countries at the beginning of the 1980s. The period mean age at first birth for the EU-15 countries rose from 24.6 years in 1975 to 28.5 years in 2001, while the period mean age of childbearing for all parities rose from 27.2 years to 29.4 years over the same period. ${ }^{2}$ In Denmark, the case study chosen here, the mean age at first birth for female cohorts rose from less than 24 years for the cohorts born immediately after World War II to over 27 years for the cohorts born in the early 1960s (see Figure 1).

The timing of childbearing is a determinate in future population size and has at least temporary effects on the age structure. To understand the effect of a change in timing on the number of births observed in a period, imagine an extreme case where in a given year, say 2007, and ever after all scheduled births were postponed by 6 months. If the originally scheduled births were evenly spaced throughout the year, then half of the births from 2007 would be postponed into the following year, cutting in half the births observed in 2007. In general, each increase in age at birth leads to a one-time decrease of births by an amount proportional to the change in mean age (Bongaarts and Feeney 1998).

Declines in births, even one-time declines, eventually reduce total population size. The rule of thumb here is that a proportional change in mean age at birth eventually reduces population size by a similar proportion. Thus, if the mean age at first birth changes from 25 to 30 , eventually population size will be about $1 / 5$ smaller than it would otherwise have been. ${ }^{3}$

2 I focus on the timing of first births in agreement with Bongaarts (2002) who writes that trends in the timing of first births are "the key factor determining trends in higher birth orders" (p. 425). The mean age of births of all orders is influenced by the parity distribution, and so parity-specific measures of timing are usually preferred.

3 This logic applies exactly when fertility is at replacement levels. For populations with non-zero intrinsic rates of natural increase, the exact effects of changing timing are more complicated. See Goldstein, Lutz and Scherbov (2003) for a discussion. 
Figure 1:

Cohort mean and standard deviation for age at first birth among Danish women, cohorts 1940-1965
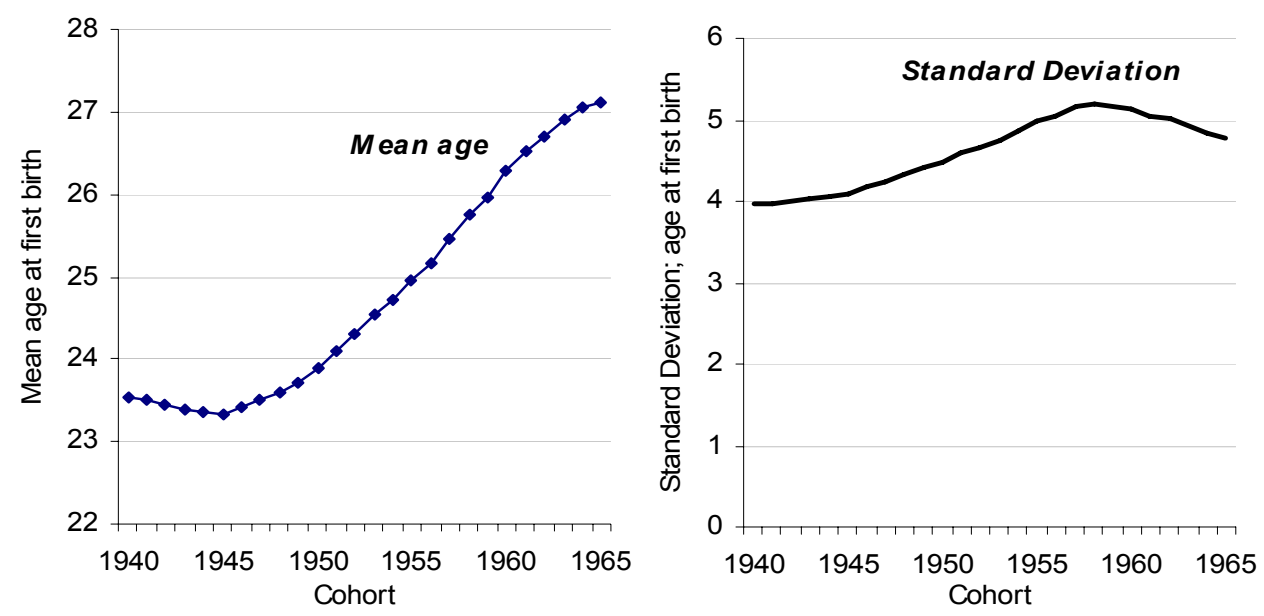

Source: Computations based on Eurostat (2006)

The population consequences of later childbearing are not just for population size but also for transient age-structure. The births that are foregone make the agestructure temporarily older, raising the long-term costs of pay-as-you-go transfer systems (Goldstein et al. 2003).

Apart from its implications for the aggregate population, the timing of childbearing is also of interest to children, parents, and grandparents. Later childbearing is frowned upon in the medical establishment because of increased health risks for mother and baby (Bewley, Davies, and Braude 2005). On the other hand, older parents may have more economic resources, lower divorce risks, and a different parenting style. Later childbearing also affects the overlap of generations. If childbearing is postponed, then grandchildren arrive later in the lives of grandparents, and parents themselves are likely to become grandparents later in life (Menken 1985).

\section{Limits to Postponement}

Clearly there are biological limits to the ages at which women can easily bear children. These may change in the future, but at present even assisted reproductive technologies decline in effectiveness as women get older. ${ }^{4}$

Reproductive technologies make it difficult to define birth timing exactly. In theory, even with today's technology, the use of frozen eggs with surrogate mothers permits having children at any age, including after death. 
It is difficult to say what the present upper limit to childbearing is. One thing that is known is that there is great heterogeneity in the ability to conceive at older ages. Leridon (2004) estimates that roughly $18 \%$ of women would not be able to have a birth after age $36,40 \%$ would not be able after age $40,90 \%$ would not be able to have a child after age 45 .

In this paper, I account for these biological limits by placing a limit of onethird on women who have a first birth after their 35th birthday. This limit is considerably less than that implied by Leridon's estimates of maximum age at last birth, which would allow one-third of women to have a child after age 42 . However, such a maximum could only be observed if every woman was aware of the oldest age at which they personally could have a child and if every woman wanted only one child. My assumptions are designed so that no great changes in the final parity distribution would be required. The upper limit used here of onethird of first births over age 35 allows most women who have a child under age 35 to have a second, and perhaps higher order, birth. Using Leridon's estimates, assuming that those who waited until after 35 were a random subset of the population with respect to their fecundity, $80 \%$ of those who are childless at age 35 could from a biological point of view still have a birth.

In addition to upper age limits stemming from biology and the heterogeneity in the effective onset of sterility, limits are also placed on the compression of the distribution of first births. Kohler, Billari and Ortega (2002) suggest that there may be "rectangularisation" of the survival curve for those remaining in the childless state that echoes the rectangularisation of the survival curve for mortality. On the other hand, there are social as well as biological reasons that such compression will stop. It is unlikely that every woman in the population will want to begin childbearing at the same time. Parity preferences differ. Educational, career, and partnership trajectories differ. And for those who try to conceive, fecundity heterogeneity will also produce a range of ages when the first birth occurs.

How far can the age at first birth be compressed? The limit I construct is that spread of the age at first birth (as measured by the standard deviation) cannot fall below that observed before postponement took place. I choose Denmark as a case study (See Figure 1). According to the cohort data gathered by Eurostat (available at www.eu.int/comm/eurostat/), the lowest standard deviation observed for a cohort was about 4.0 years for the cohorts born in the 1940s. Denmark was among those with more concentrated ages at first birth. Belgium, Spain, Finland, France, Italy, Portugal, and Sweden, never had standard deviation (SD) as low as 4.0 years. The Netherlands on the other hand had a SD of 3.8 for the cohorts born 1938 to 1945 . The UK and Norway also reached levels as low as Denmark. The most concentrated cohort fertility was seen in the former communist states, such as the GDR, which had a SDof 3.2 years in the late 1950s. 


\section{Models}

My approach to assessing the maximum age at first birth for European populations is to transform the current distribution by age at first birth in a smooth and simple way. My approach builds on the work of Kohler and Philipov (2001) remapping ages at birth and on the increasing literature on age-transformations in general (Lee and Goldstein 2003; Sanderson and Scherbov 2005). I map the share of births that occur at age $x$ in a standard baseline schedule to some new age $x^{\prime}$. In the simplest version, one can simply shift the distribution by letting the proportion of first births occurring at some original age occur at the age some constant years later. Below I consider a number of other stylised patterns for the shift, in which differential shifts occur by age. The common restriction in all of the models below is that the transformation should be monotonic in the sense that the order of ages $x_{i}^{\prime}$ is preserved in the remapped ages $x_{i}^{\prime}<x_{i}^{\prime}$. In other words, the remapped younger ages are not permitted to overtake the remapped older ages. Figure 2 illustrates some of the transformations specified below.

$a$. I begin with a baseline, or standard, age schedule $p(x)$ for the proportion of first births that occur at each age $x$. This baseline schedule is taken from the Danish cohort of 1963, the most recent birth cohort for which completed cohort fertility is available in the Eurostat database. Denmark was chosen as a population that has shown considerable postponement and where the cohort mean age at first birth has seen little slow-down in the pace of increase. ${ }^{5}$

$b$. The simplest transformation is to add some constant to each age. This is the simple shift model that is notably used by Bongaarts and Feeney (1998). It keeps the shape of the distribution but just relocates it. Formally, the shift model obeys:

$$
x^{\prime}=x+b .
$$

c. A second simple transformation is to proportionally shift each age, this is the proportional rescaling model discussed by Lee and Goldstein (2003). Under proportional rescaling,

$$
x^{\prime}=x \cdot b .
$$

(The same letter $b$ is used to represent the parameter or parameters of each transformation, but the parameter takes on different meanings and values depending on the model.) Proportional rescaling expands the variability of ages at childbearing, a trend consistent with what happened during the first decade or two of postponement, and also consistent with the greater postponement of more highly educated women (Gustafsson 2001).

5 The Eurostat database includes some imputed cohort rates at older ages. For this cohort, ages above 33 are imputed to the previous available cohort. This imputation should not have a large effect on the calculations presented here as they depend mostly on fertility rates up to the 66.6th percentile, which occurs at a considerably younger age. 
$d$. A third transformation models convergence. Recently, the variability of age at first birth has levelled off or even begun to decrease in some populations. One interpretation of this is that the behaviour of early-childbearers is beginning to follow that of late-childbearers - a kind of catching-up. The convergence of postponement can be modelled as

$$
x^{\prime}=x+\left(x_{\max }-x\right) b,
$$

This model assumes no postponement at age $x_{\max }$ and linearly increasing postponement at ages progressively younger than $x_{\max }$. This age $x_{\max }$ can either be specified in advance (e.g., age 45 or age 50), or it can be a free parameter that is selected in order to satisfy the constraints on proportion of births after age 35 and the SD. ${ }^{6}$

e. Divergence can be modelled in a parallel manner as

$$
x^{\prime}=x+\left(x-x_{\min }\right) b,
$$

in which there is no postponement at age $x_{\min }$, but postponement increases progressively at older ages. This model is consistent with the first stages of postponement in Europe, and may be relevant in the future if for example there is a resurgence of postponement among highly educated women, perhaps as a result of fertility technologies. The model might also be consistent with a population in which there is a segment of the population that does not postpone, perhaps new immigrants from early-childbearing populations or other women who prefer early childbearing

$f$. A final model I introduce is a combination of the convergence and divergence models, which I call the shifting middle. The model is

$$
x^{\prime}=x+\left(x-x_{\min }\right)\left(x_{\max }-x\right) b
$$

Here, no change occurs at the youngest and oldest ages, perhaps because of a group of non-postponers at younger ages and because of biological limits at older ages. The general form of this model is a quadratic transformation of age.

g. In addition to these models, I also present the distribution of age at birth for all parities for a late-reproducing sub-population-US women with advanced educational degrees (TFR $\approx 1.5$ ) - that has both relatively high fertility and relatively late childbearing. This population serves as an additional empirical reference point to consider. See appendix for estimation method for this population. The distribution of age at birth for all parities is shown in Figure 3.

6 The convergence model with free $x_{\max }$ is equivalent to the plastic age model $x^{\prime}=(x+m) / w$, proposed by José Antonio Ortega at the conference "Postponement of childbearing in Europe," held in Vienna, Austria, 1-3 December 2005. This formulation has the nice interpretation of a faster clock for $w>1$ and later onset for $m>0$. 
Figure 2:

Age distributions at first birth, transformations according to various models

Simple shift
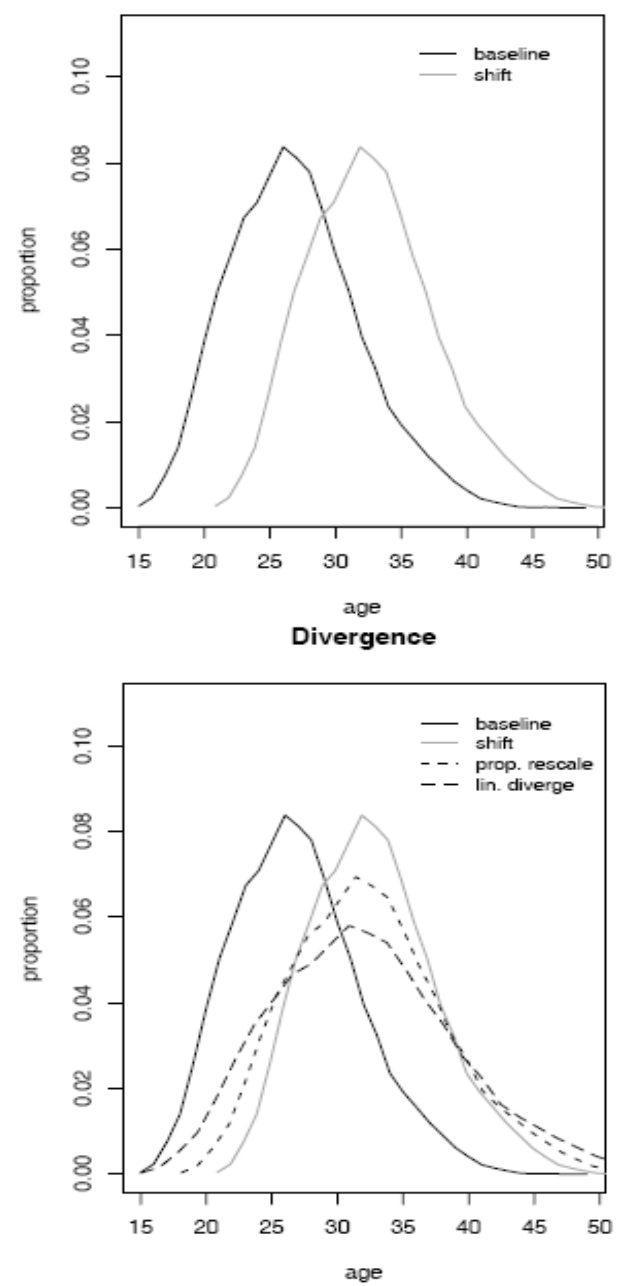

Convergence

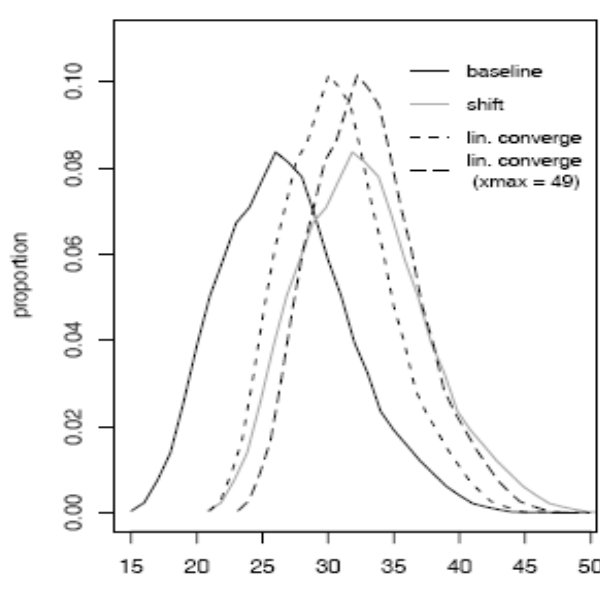

age

Shifting middle

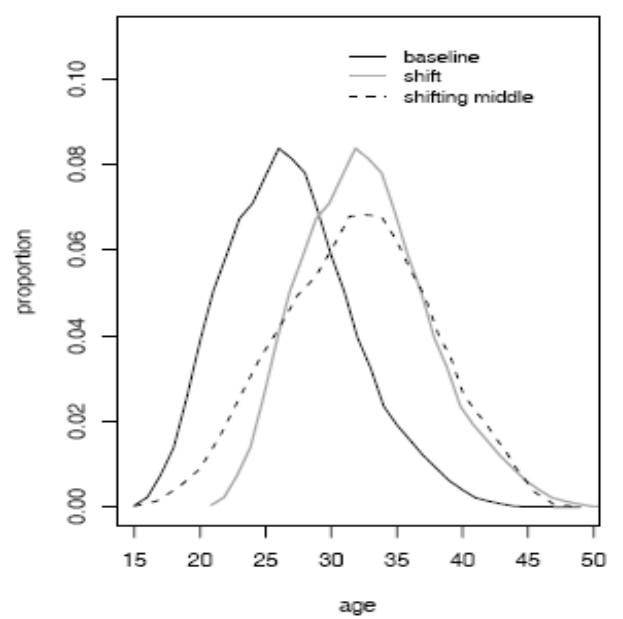

To estimate the upper limits of postponement, a search was made for the value of parameter $b$ that would make the mean of the distribution $p(x)$ as large as possible without violating the constraints on older fertility and the minimum variability of the age at first birth. For one-parameter models, I simply search through candidate values; for two-parameter models I used optimisation routines.

Alternative approaches might include the use of Brass's relational Gompertz model for fertility, which was recently applied by Yi and Land (2001) for their simulation of tempo effects, or the use of other demographic models of fertility, e.g., the Coale-Trussell model for marital fertility. 
Figure 3:

Distribution of age at first birth for Danish female cohort of 1963 (solid black) and for 1995-2000 period for US females with advanced degrees (doctors, lawyers and PhDs)

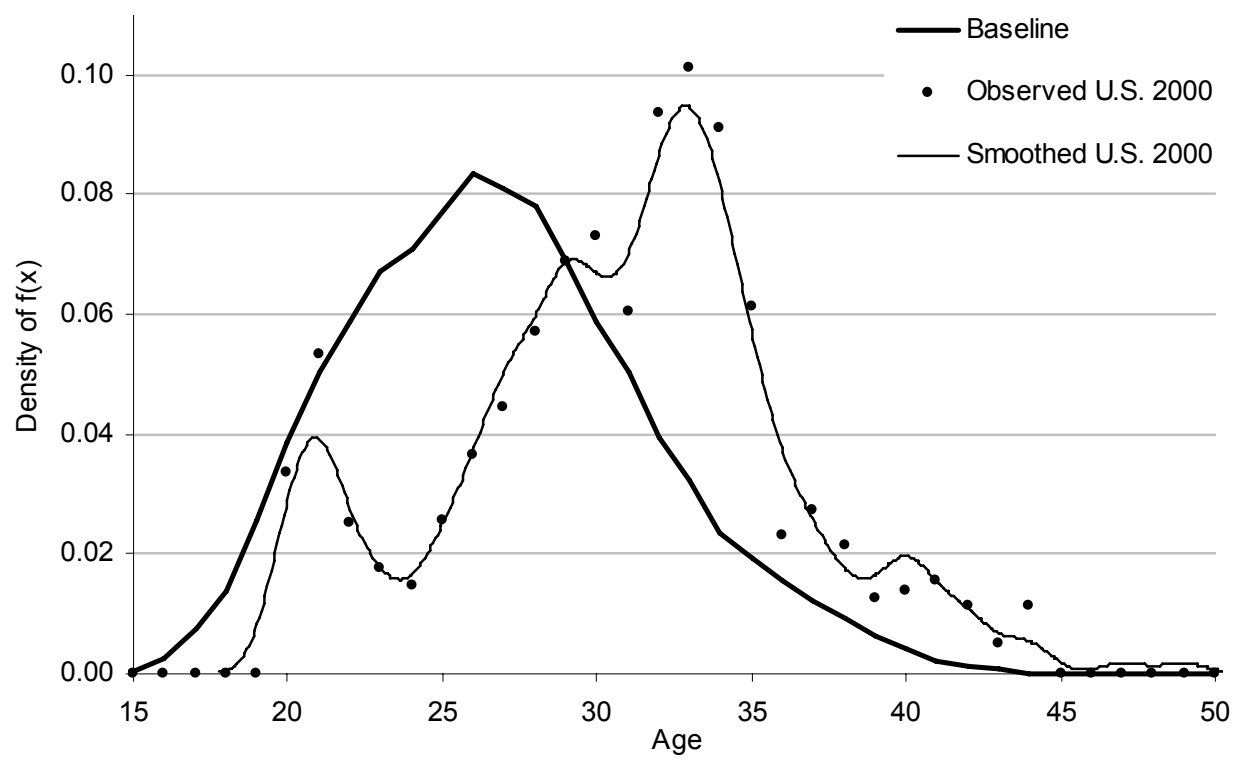

\section{Results}

The maximum mean ages according to each of the models are given in Table 1. The upper bound for the mean age according to the imposed constraints is about 33 years, roughly 6 years later than the mean age observed for the cohort of 1963 and roughly 4 years later than the period means now observed. There is clearly great room for future postponement since the baby boom postponement has moved only about half way toward the maximum.

We see that the shift model " $b$ " has nearly the highest mean (33.2 years) of all of the models, surpassed only by the linear convergence model " $e$ "" (33.4 years). The main feature of the shift model that is potentially unrealistic is the proportion of births that occur after age 40 : over $9 \%$. I say "potentially unrealistic" because it is such a contrast to current fertility patterns. However, historical "natural fertility" populations often have marital fertility rates in the 40 to 45 age group in the 0.1 to 0.2 range (Menken, Trussell and Larsen 1986), implying that the contribution to total fertility could range from 0.5 to 1.0 children, making up from a quarter to half of the births required for population replacement.

The linear convergence model reduces the proportion of births over 40 to slightly less than 7\%. In terms of early childbearing, the linear shift model is perhaps more plausible than the free linear convergence model, in which women under age 25 are responsible for only $1.4 \%$ of first births. 
Table 1:

Summary statistics of distribution of age of mother at first birth for baseline distribution, various transformed distributions, and US advanced degree subpopulation

\begin{tabular}{|c|c|c|c|c|c|c|c|}
\hline \multirow{2}{*}{ Model } & \multicolumn{2}{|c|}{ Summary Stats. } & \multicolumn{3}{|c|}{ Per cent by age } & \multicolumn{2}{|c|}{ Fitted value } \\
\hline & Mean & SD & $<25$ & $>35$ & $>40$ & $\hat{b}$ & $\hat{x}_{\max }$ \\
\hline a. Baseline & 27.3 & 4.9 & 41.3 & 7.1 & 0.9 & n.a. & n.a. \\
\hline b. Shift & 33.2 & 4.9 & 5.6 & 33.3 & 9.1 & 5.841 & n.a. \\
\hline c. Proportional Rescaling & 33.0 & 5.9 & 12.4 & 33.3 & 11.7 & 1.207 & n.a. \\
\hline d. Linear Divergence & 32.8 & 7.0 & 19.3 & 33.3 & 14.2 & 0.444 & n.a. \\
\hline $\begin{array}{l}\text { e1. Linear Convergence } \\
\left.\text { (free } x_{\max }\right)\end{array}$ & 33.3 & 4.0 & 1.4 & 33.3 & 6.7 & 0.176 & 61.4 \\
\hline $\begin{array}{l}\text { e2. Linear Convergence } \\
\left(x_{\max }=49\right)\end{array}$ & 31.1 & 4.0 & 8.4 & 17.7 & 2.6 & 0.176 & n.a. \\
\hline f. Middle Shift & 32.5 & 5.7 & 15.6 & 33.3 & 9.9 & 0.021 & n.a. \\
\hline $\begin{array}{l}\text { g. US Advanced } \\
\text { Degree } \\
\text { (*All parties) }\end{array}$ & $31.3 *$ & $5.5^{*}$ & $16.0^{*}$ & $21.0 *$ & $7.0^{*}$ & n.a. & n.a. \\
\hline
\end{tabular}

Note: Italics indicate identifying constraint.

Source: Eurostat for baseline distribution; author's calculations for transformed distributions; author's calculations from 2000 Census of the United States PUMS for US advanced-degree sup-population.

The diverging models " $c$ " and " $d$ " both give increasing standard deviations, but allow surprisingly high means of nearly 33 years before the limit on fertility over age 35 is reached. The middle shift model " $f$ " gives plausible proportions of young and old mothers, if one finds a slight increase in variation of age at first birth believable.

The very low proportions of first births under age 25 make some of the models suspect in terms of not allowing for any early childbearing. However, the recent declines in childbearing in the 15-19 age-group have been enormous. Indeed, the United States is perhaps the only country in the industrialised world with non-negligible birth rates below age 18 . One factor that may keep the proportion of births under age 25 relatively high is the presence of immigrants from early-childbearing populations.

By way of comparison, we see in Figure 3 for US women with advanced degrees that high mean ages of childbearing do not necessarily lead to extremely low total fertility. The TFR of 1.5 for these women is above that observed in many European populations, albeit may be less influenced by the pace of change in the age of childbearing (the so-called "tempo" effect). 


\section{Discussion}

The upper limits found here for the mean age at birth for cohorts are smaller than the biological upper limit. Leridon's schedule of the distribution of age at last birth in a natural fertility population implies a mean age of 44.0 with a standard deviation of 5.2 years. This distribution would be attainable only if each woman knew the oldest age at which she herself could bear a child and if each woman only wanted one child, conditions that are both quite different from reality.

I have tried to arrive at a more plausible upper limit by experimenting with a variety of simple monotonic transformations of recent cohort age schedules of first births subject to constraints. The age-schedules are transformed in such a way as to keep some fraction of births, arbitrarily chosen as two-thirds, under age 35 , and to avoid too much compression of the distribution, where "too much" is taken to be a standard deviation of 4.0 years, the level of concentration achieved by cohorts during the baby boom. These constraints were chosen arbitrarily but the hope is that they will seem plausible on their face, not as the absolute limits of fertility postponement or compression, but rather as values that are well within the realm of possibility. I can attach no probabilistic meaning to them-for example, one cannot assign a probability that these limits will be overtaken. The reason that I transform the existing schedule is that I think there is information in the shape of the schedule, its unimodality, peakedness, smoothness, etc. that reflects some of the underlying features of the distribution of future fertility in cohorts.

The "upper limits" to the population mean age at first birth are well above those observed for recent cohorts. Whereas the Danish cohort of 1963 had a mean age at first birth of 27.3 years, I find that mean ages over 33 are possible within the regime specified by my constraints. Currently, the 2004 period mean age at first birth in Denmark was 28.4 years. Postponement of first births in the EU-15 from 1975 to 2001 occurred at a rate of 1.5 years per decade. At this rate, about three decades of postponement are still possible before bumping up against the constraints I consider.

What are we to make of the levelling out that I see in some countries? For example, the period mean age at first birth in the Netherlands has not changed in several years. I don't know the reason why postponement has stopped in the Netherlands, but from the calculations presented here it appears unlikely that that country is near to the biological (or bio-social) upper limits of first birth. One explanation for the end of postponement would be that a new optimum mean age has been reached for first birth. This optimum might reflect the forces that drive postponement (e.g., educational attainment, career trajectories, desired age at marriage and so forth). ${ }^{7}$ Another explanation is that the pause in postponement is temporary and may well be resumed. ${ }^{8}$ If, as may be the case in the long run,

See Kohler et al. (2002) for a discussion of such an optimum.

Indeed, Tomáš Sobotka informs me that the most recent period data shows a further increase in age at first birth in the Netherlands. 
reproductive timing is linked to life expectancy, say through longer labour force participation, increased returns to human capital investment, etc., then the estimates calculated here can serve as upper limits with current technology.

The approach used in this paper is not meant to be exact. Could it be improved further? More insight into future variability in age at first birth could be sought, for example by looking at forecast changes in the distribution of educational attainment. Less arbitrary bounds on fertility at older ages could perhaps be obtained from looking more at the biomedical literature on fecundability or perhaps at attitudinal data in response to questions like, "What is the maximum acceptable age for women to have their first birth?" such as are being planned for future surveys.

The result that current populations are far from the upper age limits of fertility suggests the need for a new kind of demographic research. While most demographers are currently trying to understand why fertility is moving to later ages, we also need to investigate the many forces that keep fertility so early —at least compared to how late it could be.

\section{Acknowledgements}

I would like to thank Wolfgang Lutz for discussions that inspired this paper, and Tomáš Sobotka and two anonymous reviewers for useful suggestions. This research was supported in part by IIASA and VID. An earlier version of this research was presented at the conference "Postponement of childbearing in Europe", Vienna, Austria, 1-3 December 2005.

\section{References}

Bewley, S., M. Davies, and P. Braude. 2005. "Which career first?” BMJ 331(7517): 588589. «http://dx.doi.org/ 10.1 136/bmj .331.7517.588»

Bongaarts, J. 2002. "The end of the fertility transition in the developed world." Population and Development Review 28(3): 419-443.

Bongaarts, J. and G. Feeney. 1998. "On the quantum and tempo of fertility." Population and Development Review 24(2): 271-291.

Eurostat. 2006. Data on period and cohort fertility in Denmark accessed in New Cronos database (Theme 3: Population and social conditions).

«http://epp.eurostat.ec.europa.eu/portal/page?_pageid=0,1136184,0_45572595\&_dad $=$ portal\&_schema=PORTAL»

Goldstein, J. R., W. Lutz, and S. Scherbov. 2003. "Long-term population decline in Europe: The relative importance of tempo effects and generational length." Population and Development Review 29(4): 699-707. 
Gustafsson, S. 2001. "Optimal age at motherhood: Theoretical and empirical considerations on postponement of maternity in Europe." Journal of Population Economics 14: 225-247.

Hagewen, K. and S. P. Morgan. 2005. "Intended and ideal family size in the United States, 1970-2002." Population and Development Review 31(3): 507-527.

Kohler, H.-P., F. C. Billari, and J. A. Ortega. 2002. "The emergence of lowest-low fertility in Europe during the 1990s." Population and Development Review 28(4): 641-680.

Kohler, H.-P. and D. Philipov. 2001. "Variance effects in the Bongaarts-Feeney formula." Demography 38(1): 1-16.

Lee, R. and J. R. Goldstein. 2003. "Rescaling the life cycle: Longevity and proportionality." In: J. R. Carey and S. Tuljapurkar (eds.) Life Span: Evolutionary, Ecological, and Demographic Perspectives, Vol. 29 of Population and Development Review, New York: Population Council.

Leridon, H. 2004. "Can assisted reproduction technology compensate for the natural decline in fertility with age? A model assessment." Human Reproduction 19(7): 15481553.

Menken, J. 1985. “Age and fertility: how late can you wait?” Demography 22(4): 469483.

Menken, J., J. Trussell, and U. Larsen. 1986. “Age and infertility.” Science 233(4771): 1389-1394.

Sanderson, W. C. and S. Scherbov. 2005. “Average remaining lifetimes can increase as human populations age." Nature 435(7043): 811-813.

Yi, Z. and K. C. Land. 2001. "A sensitivity analysis of the Bongaarts-Feeney method for adjusting bias in observed period total fertility rates." Demography 38(1): 17-28. 


\section{Appendix A \\ Estimation of Age-Schedule of Birth for US Women with Advanced Degrees}

Fertility rates for US women with advanced degrees were calculated from the Public Use Microsample of the 2000 US census. The data files include a variable on the number of children under age 5 in the household. Fertility rates by age of woman in the household were estimated by assuming that all children under age 5 were born, on average, in 1997, 3 years prior to the census. Holders of advanced degree were defined as those with "professional" or "doctorate" degree. Those with "masters" degrees were omitted.

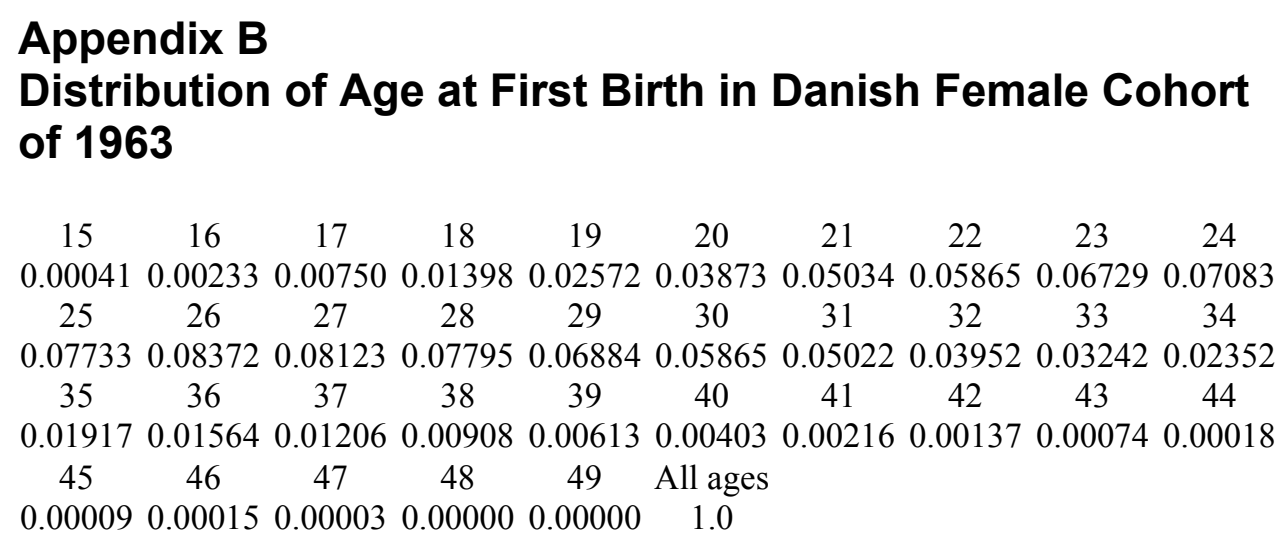

Source: Author's calculation from Eurostat. 